\title{
Emerging Role of D-Amino Acid Metabolism in the Innate Defense
}

\author{
Jumpei Sasabe* and Masataka Suzuki \\ Department of Pharmacology, School of Medicine, Keio University, Tokyo, Japan
}

Mammalian innate and adaptive immune systems use the pattern recognition receptors, such as toll-like receptors, to detect conserved bacterial and viral components. Bacteria synthesize diverse D-amino acids while eukaryotes and archaea generally produce two D-amino acids, raising the possibility that many of bacterial D-amino acids are bacteria-specific metabolites. Although D-amino acids have not been identified to bind to any known pattern recognition receptors, D-amino acids are enantioselectively recognized by some other receptors and enzymes including a flavoenzyme D-amino acid oxidase (DAO) in mammals. At host-microbe interfaces in the neutrophils and intestinal mucosa, DAO catalyzes oxidation of bacterial D-amino acids, such as D-alanine, and generates $\mathrm{H}_{2} \mathrm{O}_{2}$, which is linked to antimicrobial activity. Intestinal DAO also modifies the composition of microbiota through modulation of growth for some bacteria that are dependent on host nutrition. Furthermore, regulation and recognition of D-amino acids in mammals have additional meanings at various host-microbe interfaces; Dphenylalanine and D-tryptophan regulate chemotaxis of neutrophils through a G-coupled protein receptor, D-serine has a bacteriostatic role in the urinary tract, D-phenylalanine and D-leucine inhibit innate immunity through the sweet taste receptor in the upper airway, and D-tryptophan modulates immune tolerance in the lower airway. This minireview highlights recent evidence supporting the hypothesis that D-amino acids are utilized as inter-kingdom communication at host-microbe interface to modulate bacterial colonization and host defense.

Keywords: D-amino acid, D-amino acid oxidase, hydrogen peroxide, mucosal immunity, innate immunity, small intestine, neutrophil, host-microbe interaction

\section{INTRODUCTION}

Among all domains of life, bacteria have the largest capacity to produce wide variety of D-amino acids, whereas archaea and eukaryotes are thought to synthesize generally two kinds of D-amino acids, D-serine and D-aspartate. Bacteria utilize diverse D-amino acids in multiple biological processes to support their growth, to regulate spore germination, and to configure or remodel their cell wall (Cava et al., 2011). By contrast, mammals utilize D-serine in neurophysiology and D-aspartate in neurogenesis and endocrine systems (Fujii and Saito, 2004). Metabolism of D-amino acids in mammals involves two flavoenzymes: D-amino acid oxidase (DAO) and D-aspartate oxidase. DAO catalyzes stereoselective oxidative deamination of multiple neutral and basic D-amino acids, which yields alphaketo acids, ammonium ion, and hydrogen peroxide (Figure 1A, showing the exact chemical reaction). DAO is rarely found in bacteria, but occurs widely in most eukaryotes from yeast to humans with the exception of plants (Pollegioni et al., 2007). DAO activity 
was first described in the porcine kidney by Krebs (1935), but its physiological role was not clear because its substrates had been regarded as "unnatural" isomers of amino acids. After discovery of D-amino acids as integral components of bacterial cell wall in 1950s, Cline and Lehrer in 1969 identified DAO activity in granule fraction of human neutrophilic leukocytes (Cline and Lehrer, 1969), which is linked to bactericidal activity of leukocytes by $\mathrm{H}_{2} \mathrm{O}_{2}$ produced through oxidation of bacterial D-amino acids (Eckstein et al., 1971; DeChatelet et al., 1972). DAO has received attention by neuroscientists for past few decades because DAO in the mammalian hindbrain degrades its physiological endogenous substrate $\mathrm{D}$-serine, which binds to $N$-methyl D-aspartate (NMDA) glutamate receptors and plays crucial roles in neurophysiology and pathology (Mothet et al., 2000; Shleper et al., 2005; Sasabe et al., 2007; Basu et al., 2009; Mitchell et al., 2010; Mustafa et al., 2010; Balu et al., 2013). More recently, DAO was identified in epithelial surface of the mammalian small intestine, where interplay between mammalian DAO and bacterial D-amino acids modifies commensal bacteria and mucosal defense (Sasabe et al., 2016). Notably no mammalian genes have homology to any known bacterial genes encoding synthetic enzymes for D-amino acids, and many of bacterial D-amino acids are thought bacteriaspecific metabolites. Therefore, metabolism of bacterial D-amino acids by mammalian DAO or other molecules in the hostmicrobial interface may serve as a type of bacterial recognition.

\section{DISTRIBUTION OF DAO SUBSTRATES IN MAMMALS}

D-amino acid oxidase is distributed to the proximal tubules in the kidney with the highest expression, hepatocytes in the liver (exceptionally not detectable in mice), astrocytes in the hindbrain of the central nervous system, neutrophils, and epithelium of the small intestine (Pollegioni et al., 2007; Koga et al., 2017). Although DAO has oxidative activity selective to D-enantiomers of amino acids, DAO has a broad spectrum of substrates including multiple neutral and basic D-amino acids. In fact, mice lacking systemic DAO activity due to a missense mutation of G181R (DAO-null mice) show increased levels of various $\mathrm{D}$-amino acids, including $\mathrm{D}$-alanine, D-leucine, D-methionine, D-proline, D-phenylalanine, D-serine, and D-tyrosine, in the multiple tissues and body fluids (Koga et al., 2017). Since bacteria have the largest genetic capacity to produce wide variety of D-amino acids, most of the DAO substrates except for D-serine have been regarded to originate from bacteria. Among those D-amino acids, D-alanine and D-serine are considered major substrates of DAO in mammals because the two D-amino acids show marked increases compared to other D-amino acids in the DAO-null mice (Miyoshi et al., 2011; Koga et al., 2017).

The D-alanine and D-serine have different origins in mammals. D-Serine is produced in mammals through conversion from L-serine by an endogenous enzyme, serine racemase (SR) (Wolosker et al., 1999), expressed primarily in neurons of the central nervous system (Miya et al., 2008). The concentration of D-serine is highest (10-30\% of total serine) at submillimolar level in the forebrain (Hashimoto et al., 1992; Miyoshi et al., 2009; Suzuki et al., 2017), where D-serine serves as an endogenous coagonist with L-glutamate to activate NMDA receptors (Mothet et al., 2000; Basu et al., 2009). By contrast, DAO degrades D-serine and retains it at a low level (less than $1 \%$ of total serine) in the hindbrain and spinal cord (Miyoshi et al., 2009). In the periphery, D-serine level is usually low with the exception of that in the urine (Miyoshi et al., 2009). The D-serine is over 50\% of total urinary serine and is one of the most abundant amino acids in mammalian urine, where D-serine has a bacteriostatic role against uropathogenic bacteria (Roos and Klemm, 2006; Korte-Berwanger et al., 2013) by inhibiting L-serine metabolism and synthesis of pantothenate (Cosloy and McFall, 1973) and by modulating virulence gene expression (Roesch et al., 2003; Anfora and Welch, 2006; Anfora et al., 2007). It is also known that mammalian D-serine can repress efficient colonization of enterohaemorrhagic Escherichia coli (EHEC) by selectively inhibiting expression of a type III secretion system, which allows intimate attachment of EHEC to the host cells (Connolly et al., 2015). Such anti-bacterial D-serine is rarely synthesized in bacteria with exception of vancomycin-resistant Enterococci (Sieradzki and Tomasz, 1996; De Jonge et al., 2002; Reynolds and Courvalin, 2005) and therefore, bacteria have been thought to develop sensing system of host D-serine to recognize niche and colonize to favorable sites within the host (Connolly et al., 2016).

Another major substrate of DAO in mammals is bacterial D-alanine, one of most common D-amino acids present in the bacterial cell wall. While mammals are not capable of synthesizing D-alanine, most bacteria encode two different PLP-dependent alanine racemases, DadX and Alr, which are established drug targets for antibiotics. Both racemases catalyze the same reaction, but are components of distinct molecular pathways. In contrast to association of DadX with L-alanine catabolism (D-alanine is subsequently converted into pyruvate), Alr synthesizes the D-alanine that is utilized for peptidoglycan synthesis (Walsh, 1989; Watanabe et al., 2002). The D-alanine is incorporated into the peptides to cross-linking repeated disaccharide and provides chemical resistance to most known proteases (Nagata et al., 1998). It has become clear that intestinal $\mathrm{D}$-alanine as well as other D-amino acids including D-proline, and D-glutamate, is produced exclusively by intestinal microbiota (Sasabe et al., 2016). Furthermore, Konno et al. (1990, 1993) have shown using antibiotic-treated and germ-free (GF) mice that vast majority of $\mathrm{D}$-alanine in the serum and urine is of bacterial origin. Therefore, D-alanine produced by intestinal microbiota is, at least in part, uptaken in the intestine, circulated, and excreted into the urine. Mechanisms regulating in vivo $\mathrm{D}$-alanine kinetics, such as D-alanine transport and metabolism, still remain largely unclear.

\section{ROLE OF DAO IN THE INNATE DEFENSE BY LEUKOCYTES}

D-amino acid oxidase, conserved widely in eukaryotes but not in bacteria (Pollegioni et al., 2007), is able to generate $\mathrm{H}_{2} \mathrm{O}_{2}$ through catabolism of D-amino acids (usually of bacterial origin), and therefore, DAO has classically been considered a 

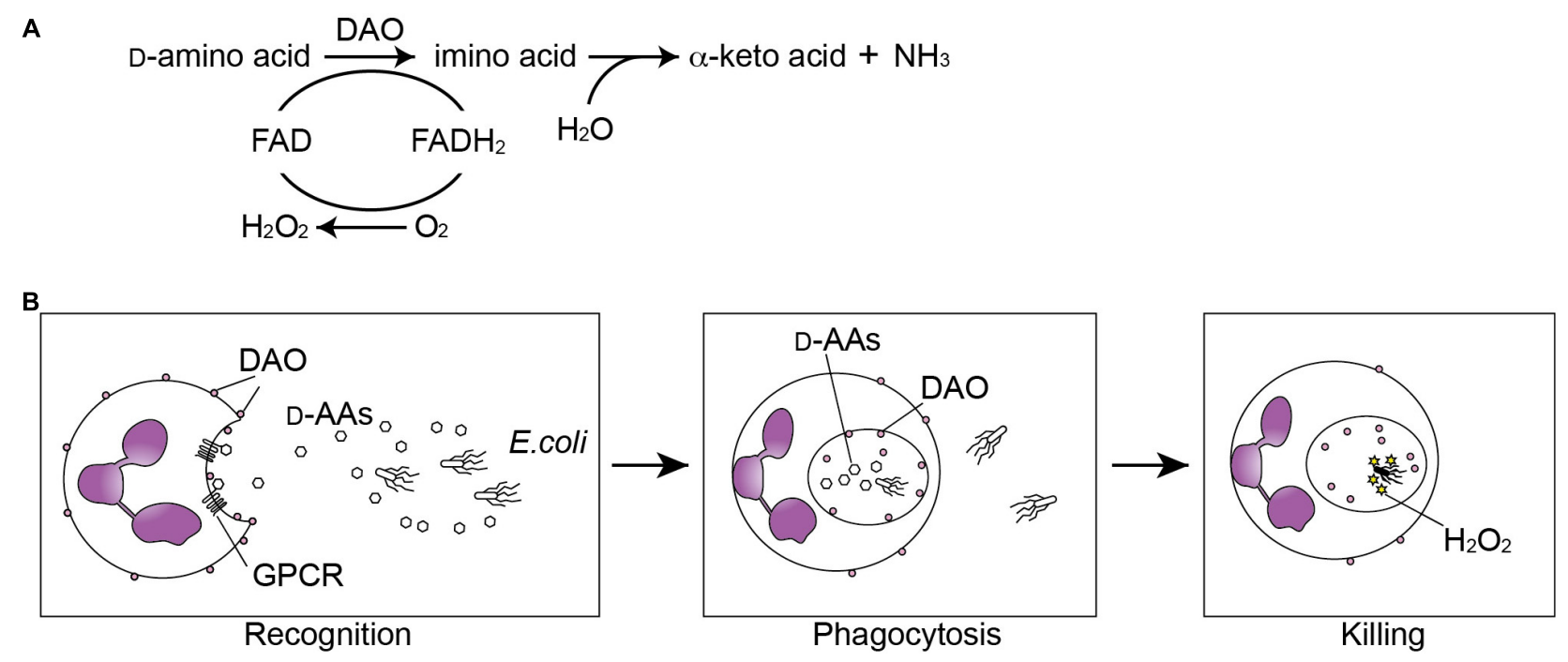

FIGURE 1 | Oxidation of D-amino acids by D-amino acid oxidase (DAO) generates anti-microbial $\mathrm{H}_{2} \mathrm{O}_{2}$. (A) DAO catalizes oxidative deamination of D-amino acids. The $\mathrm{D}$-amino acid is oxidized to an imino acid with reduction of $\mathrm{FAD}$ to $\mathrm{FADH}_{2}$, which is subsequently oxidized to $\mathrm{FAD}$ with reduction of oxygen into hydrogen peroxide. The imino acid is then non-enzymatically hydrolyzed to the corresponding alpha-keto acid and ammonia. (B) Neutrophils recognize bacterial D-amino acids through the G protein-coupled receptor (GPCR) GPR109B and are chemoattracted by bacteria. DAO oxidizes bacterial D-amino acids during the phagocytosis and generated $\mathrm{H}_{2} \mathrm{O}_{2}$ kills the bacteria.

potential component of the innate defense in mammals. Cline and Lehrer (1969) first identified DAO activity in granule fraction of guinea pig and human neutrophilic leukocytes, which is linked to bactericidal activity of leukocytes (Eckstein et al., 1971; DeChatelet et al., 1972). They have also shown in vitro that oxidation of bacterial metabolites such as D-amino acids by DAO generates $\mathrm{H}_{2} \mathrm{O}_{2}$ and subsequently activates chloride ions together with myeloperoxidase to kill E. coli. A later study using electron microscope showed that DAO is localized to the neutrophilic surface, internalized during phagocytosis, and is able to produce $\mathrm{H}_{2} \mathrm{O}_{2}$ within the phagosome (Robinson et al., 1978). On the other hand, in vivo bactericidal effect of neutrophilic DAO remains controversial. Neutrophils obtained from patients with chronic granulomatous disease (CGD), which is a primary immunodeficiency that interferes production of reactive oxygen species by phagocytes (i.e., neutrophils and macrophages) and leads to recurrent or persistent intracellular bacterial and fungal infections, have comparable DAO activity to those from control patients with bacterial infections (Eckstein et al., 1971). On the basis of the observation, it seems unlikely that oxidation of D-amino acids is the primary source of $\mathrm{H}_{2} \mathrm{O}_{2}$ generation during phagocytosis, where NADPH oxidase plays a major role (Nguyen et al., 2017). However, patients with CGD are rarely infected with catalase-negative organisms (Winkelstein et al., 2000), suggesting that a source of $\mathrm{H}_{2} \mathrm{O}_{2}$ stress independent of NADPH oxidase, such as DAO, may be selective for certain infections in these patients. It had remained unclear for more than 40 years if DAO plays a role in vivo against bacterial infection until the study using the DAO-null mice by Nakamura et al. (2012). The DAOnull mice were injected intravenously with Staphylococcus aureus, and showed increased number of the bacteria in the kidney and reduced survival rate compared to wild-type controls. However, they did not find ex vivo bactericidal effect of DAO in neutrophils derived from peritoneal cavity against $S$. aureus, warranting further examination using conditional knockout to unveil the physiological role of neutrophilic DAO. Another in vivo study has shown that neutrophil DAO functions to exert bactericidal activity on intraperitoneally injected Salmonella typhimurium at early stages of infection (Tuinema et al., 2014), when neutrophils are the major cell type infected by Salmonella (Loetscher et al., 2012). Interestingly, S. typhimurium limits exposure to oxidative damage elicited by DAO through importing D-alanine by dalS, an $\mathrm{ABC}$ importer specific for D-alanine. Indeed, dalS mutants of S. typhimurium are exposed to greater $\mathrm{H}_{2} \mathrm{O}_{2}$ stress than the wild type in vivo, which is attenuated by the presence of a chemical DAO inhibitor, 6-chloro-1,2-benzisoxazol-3(2H)-one (CBIO) (Tuinema et al., 2014). On the other hand, aromatic $\mathrm{D}$-amino acids, such as D-phenylalanine and D-tryptophan, act as chemoattractant factors for human leukocytes through a $G$ protein-coupled receptor, GPR109B (Irukayama-Tomobe et al., 2009). Collectively, these studies imply a mechanistic insight into a host-pathogen interaction; neutrophils are chemo-attracted by bacterial $\mathrm{D}$-amino acids and kill bacteria through oxidation of the amino acids in phagosome (Figure 1B), while bacteria evade bactericidal activity of DAO through actively importing its substrates.

\section{DAO AS AN ANTI-MICROBIAL FACTOR IN THE MUCOSAL INNATE DEFENSE}

In addition to the bactericidal function of neutrophilic DAO, regulation of $\mathrm{D}$-amino acids by $\mathrm{DAO}$ has recently been associated with mucosal homeostasis (Sasabe et al., 2016). DAO activity 

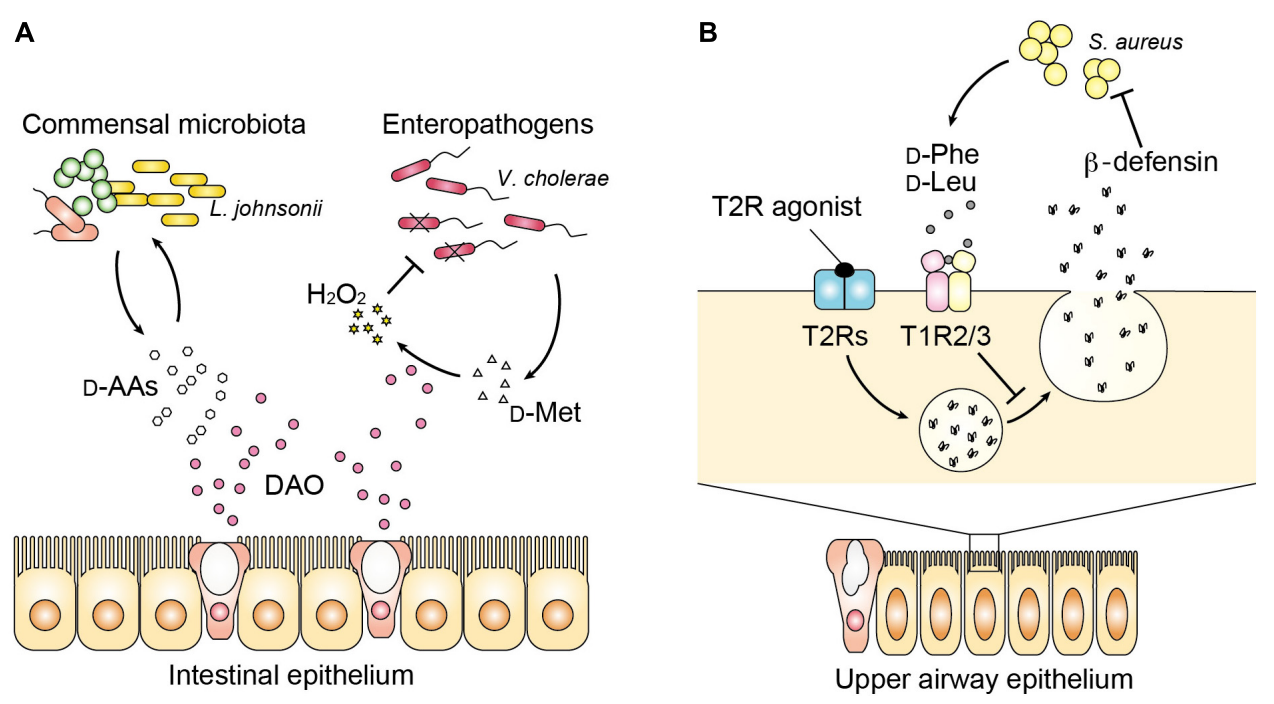

FIGURE 2 | Host-microbe communication with D-amino acids in the mucosa. (A) DAO influences luminal bacteria in the intestinal mucosa with bimodal functions. DAO modifies the composition of commensal microbiota partly by modulating availability of D-amino acids for bacterial growth. On the other hand, DAO limits colonization of enteropathogens such as Vibrio cholerae by generation of $\mathrm{H}_{2} \mathrm{O}_{2}$ through oxidation of bacterial $\mathrm{D}$-amino acids. (B) In the upper airway, bacterial D-phenylalanine and D-leucine bind to the sweet taste receptor (T1R2/3). Release of antimicrobial peptides including beta-defensin by activation of the bitter taste receptor (T2Rs) is suppressed by signaling from the sweet taste receptor.

was reported in luminal epithelium of the small intestine in fish (Sarower et al., 2003a,b), chickens (Brachet and Puigserver, 1992), mice and humans (Sasabe et al., 2016). Intestinal DAO was described in common carp as a metabolizing agent for $\mathrm{D}$-alanine because free $\mathrm{D}$-alanine is abundant exceptionally in aquatic invertebrates, besides bacteria, such as crustaceans and bivalve mollusks (D'Aniello and Giuditta, 1980; Matsushima et al., 1984), which are potential food sources of these fish. Of note, feeding carps with D-alanine increases intestinal DAO activity by eightfold, suggesting that inducible nature of fish $\mathrm{DAO}$ is associated with its role in metabolizing exogenous D-alanine (Sarower et al., 2003b). Distribution of intestinal $\mathrm{DAO}$ in chickens shows similar pattern as that in mice. In chicken and mice, DAO activity is detected in the mucosa of small intestine and higher in the proximal part compared to the distal part within small intestine (Brachet and Puigserver, 1992; Sasabe et al., 2016). Importantly, intestinal DAO in mice and humans localizes to Muc2-positive secretory vesicles of goblet cells as well as to enterocytes, which is inducible by the presence of vancomycin-sensitive intestinal microbiota, and oxidizes DAO substrates such as D-alanine in the mucosa and epithelium (Sasabe et al., 2016). Therefore, host DAO is induced and released in response to certain intestinal microbiota to react to microbial metabolites. Such microbe-host interaction can affect intestinal microbiota presumably in two ways (Sasabe et al., 2016) (Figure 2A). The first is the similar way as described in the leukocytes that oxidation of bacterial D-amino acids generates bactericidal $\mathrm{H}_{2} \mathrm{O}_{2}$, which limits colonization of pathogenic bacteria including Vibrio cholerae in the small intestine. $V$. cholerae and $V$. parahaemolyticus are more sensitive to DAO than Listeria monocytogenes, EHEC, or Salmonella enterica (Sasabe et al., 2016), in part, because Vibrio produces a good substrate of DAO D-methionine by expressing a broad spectrum amino acid racemase in Vibrio (bsrV) (Lam et al., 2009). On the other hand, commensal bacteria Lactobacilli are resistant to $\mathrm{H}_{2} \mathrm{O}_{2}$ (Serata et al., 2012) and not killed by DAO. Therefore, bactericidal activity of DAO may be defined by bacterial production/release of DAO substrates and resistance to $\mathrm{H}_{2} \mathrm{O}_{2}$ stress. The second is associated with nutritional niche for certain bacteria that are dependent on host nutrients and receive benefit from $\mathrm{D}$-amino acids for their growth, which modifies the composition of the commensal microbiota. For example, loss of DAO results in increase of Lactobacillus johnsonii (Sasabe et al., 2016), which completely lacks genes encoding biosynthetic pathways for amino acids (Pridmore et al., 2004) and obtains growth support by D-alanine (van der Kaaij et al., 2004). Furthermore, of note, loss of such mucosal homeostasis by DAO increases the level of secretory IgA in the feces (Sasabe et al., 2016). Thus, these findings identify previously unrecognized physiological role of metabolizing $\mathrm{D}$-amino acid by intestinal DAO in mucosal immunity.

\section{MODIFICATION OF MUCOSAL IMMUNITY BY BACTERIAL D-AMINO ACIDS BEYOND DAO}

In the upper respiratory airway, it has become clear that some D-amino acids modify innate immunity although involvement of DAO has not been studied yet. Human saliva contains substantial amount of free $\mathrm{D}$-amino acids such as $\mathrm{D}$-alanine ( $\sim 30 \%$ of total alanine), D-proline ( $\sim 20 \%$ of total proline), and D-aspartate $(\sim 10 \%$ of total aspartate) (Battistone and Burnett, 1961; Syrjanen et al., 1990; Nagata et al., 2006). The whole chiral 
profile of salivary amino acid remains uncertain, but more kinds of D-amino acids may exist considering their diverse origins including food and oral commensal microbiota. Whereas most L-amino acids are known to taste bitter, D-amino acids taste usually sweet presumably because of stereoselectivity of sweet taste receptors (T1R2/3), which preferentially bind the D-amino acids including D-tryptophan, D-phenylalanine, D-leucine, and D-histidine (Bassoli et al., 2014). Interestingly, sweet and bitter taste receptors present in the upper airway are known to influence antimicrobial innate immune responses. Activation of bitter taste receptors (T2Rs) stimulates surrounding epithelial cells to release antimicrobial peptides (Lee et al., 2014), but the sweet taste receptor (T1R) inhibits this response (Margolskee, 2002). Two D-amino acids (D-leucine and D-phenylalanine), found in respiratory isolates of Staphylococcus species inhibit the release of antimicrobial peptides by activating $\mathrm{T} 1 \mathrm{R} 2 / 3$, and increase cell death of human sinonasal epithelial culture in response to infection with methicillin-resistant $S$. aureus (Lee et al., 2017) (Figure 2B). Thus, D-amino acids produced by nasal microbiota can inhibit innate immune response through sweet taste receptors and may shape the microbial community of the upper airways. Kepert et al. (2017) have shown further evidence to support the role of bacterial D-amino acids in the mucosal immunity both in the lower airway and intestine. They identified D-tryptophan, screened from supernatants of probiotic bacteria, to reduce secretion of chemokine ligand 17 (CCL17) in a human Hodgkin lymphoma T-cell line and to induce IL10 and decrease LPS-induced IFN-gamma, IL-12, and IL-5 in human monocyte-derived dendritic cells. Oral supplementation of D-tryptophan in mice alters diversity of gut microbiota, increases numbers of regulatory $\mathrm{T}$ cells in the lung and colon, decreases lung Th2 responses, and ameliorates allergic airway inflammation and hyperresponsiveness (Kepert et al., 2017). Although further mechanisms underlying connection between innate and acquired immunity modified by bacterial D-amino acids remain largely unknown, recognition of bacterial D-amino acids by the mammalian enzyme or receptors may play a significant role in the mucosal immunity and homeostasis.

\section{REFERENCES}

Anfora, A. T., Haugen, B. J., Roesch, P., Redford, P., and Welch, R. A. (2007). Roles of serine accumulation and catabolism in the colonization of the murine urinary tract by Escherichia coli CFT073. Infect. Immun. 75, 5298-5304. doi: 10.1128/IAI.00652-07

Anfora, A. T., and Welch, R. A. (2006). DsdX is the second D-serine transporter in uropathogenic Escherichia coli clinical isolate CFT073. J. Bacteriol. 188, 6622-6628. doi: 10.1128/JB.00634-06

Balu, D. T., Li, Y., Puhl, M. D., Benneyworth, M. A., Basu, A. C., Takagi, S., et al. (2013). Multiple risk pathways for schizophrenia converge in serine racemase knockout mice, a mouse model of NMDA receptor hypofunction. Proc. Natl. Acad. Sci. U.S.A. 110, E2400-E2409. doi: 10.1073/pnas.1304308110

Bassoli, A., Borgonovo, G., Caremoli, F., and Mancuso, G. (2014). The taste of Dand L-amino acids: In vitro binding assays with cloned human bitter (TAS2Rs) and sweet (TAS1R2/TAS1R3) receptors. Food Chem. 150, 27-33. doi: 10.1016/j. foodchem.2013.10.106

Basu, A. C., Tsai, G. E., Ma, C. L., Ehmsen, J. T., Mustafa, A. K., Han, L., et al. (2009). Targeted disruption of serine racemase affects glutamatergic neurotransmission and behavior. Mol. Psychiatry 14, 719-727. doi: 10.1038/mp.2008.130

\section{CONCLUSION}

In mammals, intrinsic $\mathrm{D}$-serine and $\mathrm{D}$-aspartate have received great attention for their neuromodulatory roles in the central nervous system. In this mini-review, we have shed light on previously less-focused D-amino acids originated from commensal or pathogenic bacteria. As bacteria produce and release a largely distinct set of $\mathrm{D}$-amino acids from mammals, accumulating evidence show that bacterial D-amino acids serve as inter-kingdom signals linked to innate defense in mammals. At the host-microbe interfaces, host reacts to bacteria through enantioselective recognition of $\mathrm{D}$-amino acids by $\mathrm{DAO}$ or sweet taste receptors, and provides direct toxic response or indirect actions through modulating antimicrobial peptides. Furthermore, such recognition of bacterial D-amino acids by host may further mediate signals to modulate adaptive immunity.

\section{AUTHOR CONTRIBUTIONS}

JS and MS contributed to the planning and writing of the manuscript.

\section{FUNDING}

JS was funded by JSPS KAKENHI Grant Number 16K09327, Moritani Scholarship Foundation, and Keio Gijuku Fukuzawa Memorial Fund for the Advancement of Education and Research. MS was supported by JSPS KAKENHI Grant Number 18K07181 and Grant-in-Aid for JSPS Research Fellow (Grant Number 17J10213).

\section{ACKNOWLEDGMENTS}

We thank M. Yasui for indispensable support.

Battistone, G. C., and Burnett, G. W. (1961). The free amino acid composition of human saliva. Arch. Oral. Biol. 3, 161-170. doi: 10.1016/0003-9969(61)90133-9

Brachet, P., and Puigserver, A. (1992). Regional differences for the D-amino acid oxidase-catalysed oxidation of D-methionine in chicken small intestine. Comp. Biochem. Physiol. B 101, 509-511. doi: 10.1016/0305-0491(92) 90329-P

Cava, F., Lam, H., de Pedro, M. A., and Waldor, M. K. (2011). Emerging knowledge of regulatory roles of D-amino acids in bacteria. Cell. Mol. Life Sci. 68, 817-831. doi: 10.1007/s00018-010-0571-8

Cline, M. J., and Lehrer, R. I. (1969). D-amino acid oxidase in leukocytes: a possible D-amino-acid-linked antimicrobial system. Proc. Natl. Acad. Sci. U.S.A. 62, 756-763. doi: 10.1073/pnas.62.3.756

Connolly, J. P., Gabrielsen, M., Goldstone, R. J., Grinter, R., Wang, D., Cogdell, R. J., et al. (2016). A highly conserved bacterial D-serine uptake system links host metabolism and virulence. PLoS Pathog. 12:e1005359. doi: 10.1371/journal. ppat.1005359

Connolly, J. P., Goldstone, R. J., Burgess, K., Cogdell, R. J., Beatson, S. A., Vollmer, W., et al. (2015). The host metabolite D-serine contributes to bacterial niche specificity through gene selection. ISME J. 9, 1039-1051. doi: 10.1038/ ismej.2014.242 
Cosloy, S. D., and McFall, E. (1973). Metabolism of D-serine in Escherichia coli K-12: mechanism of growth inhibition. J. Bacteriol. 114, 685-694.

D'Aniello, A., and Giuditta, A. (1980). Presence of D-alanine in crustacean muscle and hepatopancreas. Comp. Biochem. Physiol. B 66, 319-322. doi: 10.1016/03050491(80)90071-1

De Jonge, B. L., Gage, D., and Xu, N. (2002). The carboxyl terminus of peptidoglycan stem peptides is a determinant for methicillin resistance in Staphylococcus aureus. Antimicrob. Agents Chemother. 46, 3151-3155. doi: 10.1128/AAC.46.10.3151-3155.2002

DeChatelet, L. R., McCall, C. E., and Cooper, M. R. (1972). Amino acid oxidase in leukocytes: evidence against a major role in phagocytosis. Infect. Immun. 5, 632-633.

Eckstein, M. R., Baehner, R. L., and Nathan, D. G. (1971). Amino acid oxidase of leukocytes in relation to $\mathrm{H}_{2} \mathrm{O}_{2}$-mediated bacterial killing. J. Clin. Invest. 50, 1985-1991. doi: 10.1172/JCI106690

Fujii, N., and Saito, T. (2004). Homochirality and life. Chem. Rec. 4, 267-278. doi: $10.1002 /$ tcr. 20020

Hashimoto, A., Nishikawa, T., Hayashi, T., Fujii, N., Harada, K., Oka, T., et al. (1992). The presence of free D-serine in rat brain. FEBS Lett. 296, 33-36. doi: 10.1016/0014-5793(92)80397-Y

Irukayama-Tomobe, Y., Tanaka, H., Yokomizo, T., Hashidate-Yoshida, T., Yanagisawa, M., and Sakurai, T. (2009). Aromatic D-amino acids act as chemoattractant factors for human leukocytes through a $\mathrm{G}$ protein-coupled receptor, GPR109B. Proc. Natl. Acad. Sci. U.S.A. 106, 3930-3934. doi: 10.1073/ pnas.0811844106

Kepert, I., Fonseca, J., Muller, C., Milger, K., Hochwind, K., Kostric, M., et al. (2017). D-tryptophan from probiotic bacteria influences the gut microbiome and allergic airway disease. J. Allergy Clin. Immunol. 139, 1525-1535. doi: 10.1016/j.jaci.2016.09.003

Koga, R., Miyoshi, Y., Sakaue, H., Hamase, K., and Konno, R. (2017). Mouse D-amino-acid oxidase: distribution and physiological substrates. Front. Mol. Biosci. 4:82. doi: 10.3389/fmolb.2017.00082

Konno, R., Niwa, A., and Yasumura, Y. (1990). Intestinal bacterial origin of D-alanine in urine of mutant mice lacking D-amino-acid oxidase. Biochem. J. 268, 263-265. doi: 10.1042/bj2680263

Konno, R., Oowada, T., Ozaki, A., Iida, T., Niwa, A., Yasumura, Y., et al. (1993). Origin of D-alanine present in urine of mutant mice lacking D-amino-acid oxidase activity. Am. J. Physiol. 265(4 Pt 1), G699-G703. doi: 10.1152/ajpgi. 1993.265.4.G699

Korte-Berwanger, M., Sakinc, T., Kline, K., Nielsen, H. V., Hultgren, S., and Gatermann, S. G. (2013). Significance of the D-serine-deaminase and D-serine metabolism of Staphylococcus saprophyticus for virulence. Infect. Immun. 81, 4525-4533. doi: 10.1128/IAI.00599-13

Krebs, H. A. (1935). Metabolism of amino-acids: deamination of amino-acids. Biochem. J. 29, 1620-1644. doi: 10.1042/bj0291620

Lam, H., Oh, D. C., Cava, F., Takacs, C. N., Clardy, J., de Pedro, M. A., et al. (2009). $\mathrm{D}$-amino acids govern stationary phase cell wall remodeling in bacteria. Science 325, 1552-1555. doi: 10.1126/science. 1178123

Lee, R. J., Hariri, B. M., McMahon, D. B., Chen, B., Doghramji, L., Adappa, N. D., et al. (2017). Bacterial d-amino acids suppress sinonasal innate immunity through sweet taste receptors in solitary chemosensory cells. Sci. Signal. 10:eaam7703. doi: 10.1126/scisignal.aam7703

Lee, R. J., Kofonow, J. M., Rosen, P. L., Siebert, A. P., Chen, B., Doghramji, L., et al. (2014). Bitter and sweet taste receptors regulate human upper respiratory innate immunity. J. Clin. Invest. 124, 1393-1405. doi: 10.1172/JCI72094

Loetscher, Y., Wieser, A., Lengefeld, J., Kaiser, P., Schubert, S., Heikenwalder, M., et al. (2012). Salmonella transiently reside in luminal neutrophils in the inflamed gut. PLoS One 7:e34812. doi: 10.1371/journal.pone.0034812

Margolskee, R. F. (2002). Molecular mechanisms of bitter and sweet taste transduction. J. Biol. Chem. 277, 1-4. doi: 10.1074/jbc.R100054200

Matsushima, O., Katayama, H., Yamada, K., and Kado, Y. (1984). Occurrence of free $\mathrm{D}$-alanine and alanine racemase activity in bivalve molluscs with special reference to intracellular osmoregulation. Mar. Biol. Lett. 5, 217-225.

Mitchell, J., Paul, P., Chen, H. J., Morris, A., Payling, M., Falchi, M., et al. (2010). Familial amyotrophic lateral sclerosis is associated with a mutation in D-amino acid oxidase. Proc. Natl. Acad. Sci. U.S.A. 107, 7556-7561. doi: 10.1073/pnas. 0914128107
Miya, K., Inoue, R., Takata, Y., Abe, M., Natsume, R., Sakimura, K., et al. (2008). Serine racemase is predominantly localized in neurons in mouse brain. J. Comp. Neurol. 510, 641-654. doi: 10.1002/cne.21822

Miyoshi, Y., Hamase, K., Okamura, T., Konno, R., Kasai, N., Tojo, Y., et al. (2011). Simultaneous two-dimensional HPLC determination of free D-serine and D-alanine in the brain and periphery of mutant rats lacking D-amino-acid oxidase. J. Chromatogr. B Analyt. Technol. Biomed. Life Sci. 879, 3184-3189. doi: 10.1016/j.jchromb.2010.08.024

Miyoshi, Y., Hamase, K., Tojo, Y., Mita, M., Konno, R., and Zaitsu, K. (2009). Determination of $\mathrm{D}$-serine and $\mathrm{D}$-alanine in the tissues and physiological fluids of mice with various D-amino-acid oxidase activities using twodimensional high-performance liquid chromatography with fluorescence detection. J. Chromatogr. B Analyt. Technol. Biomed. Life Sci. 877, 2506-2512. doi: 10.1016/j.jchromb.2009.06.028

Mothet, J. P., Parent, A. T., Wolosker, H., Brady, R. O. Jr., Linden, D. J., Ferris, C. D., et al. (2000). D-serine is an endogenous ligand for the glycine site of the N-methyl-D-aspartate receptor. Proc. Natl. Acad. Sci. U.S.A. 97, 4926-4931. doi: $10.1073 /$ pnas. 97.9 .4926

Mustafa, A. K., Ahmad, A. S., Zeynalov, E., Gazi, S. K., Sikka, G., Ehmsen, J. T., et al. (2010). Serine racemase deletion protects against cerebral ischemia and excitotoxicity. J. Neurosci. 30, 1413-1416. doi: 10.1523/JNEUROSCI.4297-09. 2010

Nagata, Y., Fujiwara, T., Kawaguchi-Nagata, K., Fukumori, Y., and Yamanaka, T. (1998). Occurrence of peptidyl D-amino acids in soluble fractions of several eubacteria, archaea and eukaryotes. Biochim. Biophys. Acta 1379, 76-82. doi: 10.1016/S0304-4165(97)00084-6

Nagata, Y., Higashi, M., Ishii, Y., Sano, H., Tanigawa, M., Nagata, K., et al. (2006). The presence of high concentrations of free $\mathrm{D}$-amino acids in human saliva. Life Sci. 78, 1677-1681. doi: 10.1016/j.lfs.2005.08.009

Nakamura, H., Fang, J., and Maeda, H. (2012). Protective role of D-amino acid oxidase against Staphylococcus aureus infection. Infect. Immun. 80, 1546-1553. doi: 10.1128/IAI.06214-11

Nguyen, G. T., Green, E. R., and Mecsas, J. (2017). Neutrophils to the ROScue: mechanisms of NADPH oxidase activation and bacterial resistance. Front. Cell. Infect. Microbiol. 7:373. doi: 10.3389/fcimb.2017.00373

Pollegioni, L., Piubelli, L., Sacchi, S., Pilone, M. S., and Molla, G. (2007). Physiological functions of D-amino acid oxidases: from yeast to humans. Cell. Mol. Life Sci. 64, 1373-1394. doi: 10.1007/s00018-007-6558-4

Pridmore, R. D., Berger, B., Desiere, F., Vilanova, D., Barretto, C., Pittet, A. C., et al. (2004). The genome sequence of the probiotic intestinal bacterium Lactobacillus johnsonii NCC 533. Proc. Natl. Acad. Sci. U.S.A. 101, 2512-2517. doi: 10.1073/ pnas. 0307327101

Reynolds, P. E., and Courvalin, P. (2005). Vancomycin resistance in enterococci due to synthesis of precursors terminating in D-alanyl-D-serine. Antimicrob. Agents Chemother. 49, 21-25. doi: 10.1128/AAC.49.1.21-25.2005

Robinson, J. M., Briggs, R. T., and Karnovsky, M. J. (1978). Localization of D-amino acid oxidase on the cell surface of human polymorphonuclear leukocytes. J. Cell Biol. 77, 59-71. doi: 10.1083/jcb.77.1.59

Roesch, P. L., Redford, P., Batchelet, S., Moritz, R. L., Pellett, S., Haugen, B. J., et al. (2003). Uropathogenic Escherichia coli use d-serine deaminase to modulate infection of the murine urinary tract. Mol. Microbiol. 49, 55-67. doi: 10.1046/j. 1365-2958.2003.03543.x

Roos, V., and Klemm, P. (2006). Global gene expression profiling of the asymptomatic bacteriuria Escherichia coli strain 83972 in the human urinary tract. Infect. Immun. 74, 3565-3575. doi: 10.1128/IAI.01959-05

Sarower, M. G., Matsui, T., and Abe, H. (2003a). Distribution and characteristics of D-amino acid and D-aspartate oxidases in fish tissues. J. Exp. Zool. A Comp. Exp. Biol. 295, 151-159. doi: 10.1002/jez.a.10217

Sarower, M. G., Okada, S., and Abe, H. (2003b). Molecular characterization of $\mathrm{D}$-amino acid oxidase from common carp Cyprinus carpio and its induction with exogenous free D-alanine. Arch. Biochem. Biophys. 420, 121-129.

Sasabe, J., Chiba, T., Yamada, M., Okamoto, K., Nishimoto, I., Matsuoka, M., et al. (2007). D-serine is a key determinant of glutamate toxicity in amyotrophic lateral sclerosis. EMBO J. 26, 4149-4159. doi: 10.1038/sj.emboj.7601840

Sasabe, J., Miyoshi, Y., Rakoff-Nahoum, S., Zhang, T., Mita, M., Davis, B. M., et al. (2016). Interplay between microbial d-amino acids and host d-amino acid oxidase modifies murine mucosal defence and gut microbiota. Nat. Microbiol. 1:16125. doi: 10.1038/nmicrobiol.2016.125 
Serata, M., Iino, T., Yasuda, E., and Sako, T. (2012). Roles of thioredoxin and thioredoxin reductase in the resistance to oxidative stress in Lactobacillus casei. Microbiology 158(Pt 4), 953-962. doi: 10.1099/mic.0.05 3942-0

Shleper, M., Kartvelishvily, E., and Wolosker, H. (2005). D-serine is the dominant endogenous coagonist for NMDA receptor neurotoxicity in organotypic hippocampal slices. J. Neurosci. 25, 9413-9417. doi: 10.1523/JNEUROSCI.319005.2005

Sieradzki, K., and Tomasz, A. (1996). A highly vancomycin-resistant laboratory mutant of Staphylococcus aureus. FEMS Microbiol. Lett. 142, 161-166. doi: 10.1111/j.1574-6968.1996.tb08424.x

Suzuki, M., Imanishi, N., Mita, M., Hamase, K., Aiso, S., and Sasabe, J. (2017). Heterogeneity of D-serine distribution in the human central nervous system. ASN Neuro 9:1759091417713905. doi: 10.1177/17590914177 13905

Syrjanen, S. M., Alakuijala, L., Alakuijala, P., Markkanen, S. O., and Markkanen, H. (1990). Free amino acid levels in oral fluids of normal subjects and patients with periodontal disease. Arch. Oral. Biol. 35, 189-193. doi: 10.1016/0003-9969(90) 90054-E

Tuinema, B. R., Reid-Yu, S. A., and Coombes, B. K. (2014). Salmonella evades D-amino acid oxidase to promote infection in neutrophils. mBio 5:e01886-14. doi: 10.1128/mBio.01886-14

van der Kaaij, H., Desiere, F., Mollet, B., and Germond, J. E. (2004). L-alanine auxotrophy of Lactobacillus johnsonii as demonstrated by physiological, genomic, and gene complementation approaches. Appl. Environ. Microbiol. 70, 1869-1873. doi: 10.1128/AEM.70.3.1869-1873.2004
Walsh, C. T. (1989). Enzymes in the D-alanine branch of bacterial cell wall peptidoglycan assembly. J. Biol. Chem. 264, 2393-2396.

Watanabe, A., Yoshimura, T., Mikami, B., Hayashi, H., Kagamiyama, H., and Esaki, N. (2002). Reaction mechanism of alanine racemase from Bacillus stearothermophilus: $\mathrm{x}$-ray crystallographic studies of the enzyme bound with N(5'-phosphopyridoxyl)alanine. J. Biol. Chem. 277, 19166-19172. doi: 10.1074/ jbc.M201615200

Winkelstein, J. A., Marino, M. C., Johnston, R. B. Jr., Boyle, J., Curnutte, J., Gallin, J. I., et al. (2000). Chronic granulomatous disease. Report on a national registry of 368 patients. Medicine 79, 155-169. doi: 10.1097/00005792-20000500000003

Wolosker, H., Sheth, K. N., Takahashi, M., Mothet, J. P., Brady, R. O. Jr., Ferris, C. D., et al. (1999). Purification of serine racemase: biosynthesis of the neuromodulator D-serine. Proc. Natl. Acad. Sci. U.S.A. 96, 721-725. doi: $10.1073 /$ pnas.96.2.721

Conflict of Interest Statement: The authors declare that the research was conducted in the absence of any commercial or financial relationships that could be construed as a potential conflict of interest.

Copyright (c) 2018 Sasabe and Suzuki. This is an open-access article distributed under the terms of the Creative Commons Attribution License (CC BY). The use, distribution or reproduction in other forums is permitted, provided the original author(s) and the copyright owner are credited and that the original publication in this journal is cited, in accordance with accepted academic practice. No use, distribution or reproduction is permitted which does not comply with these terms. 